\title{
Use of the single-image approach in multiple-image deblurring problems with Poissonian noise
}

\author{
R. Vio ${ }^{1}$ and W. Wamsteker ${ }^{2}$ \\ ${ }^{1}$ Chip Computers Consulting s.r.l., Viale Don L. Sturzo 82, S. Liberale di Marcon, 30020 Venice, Italy \\ e-mail: robertovio@tin.it \\ 2 ESA-VILSPA, Apartado 50727, 28080 Madrid, Spain \\ e-mail: willem.wamsteker@esa.int
}

Received 27 September 2004 / Accepted 1 June 2005

\begin{abstract}
It has been shown that the restoration of a set of images of a fixed object, blurred by different space-invariant point spread functions (PFS) and contaminated by additive Gaussian noise, can be reformulated as a standard single-image leastsquares (LS) deblurring problem with remarkable advantages that concern both the computational burden as well as the storage memory. The only difficulty is represented by the fact that the resulting single-image can have negative values. In a recent paper by Anconelli et al. (2005, A\&A, 430, 731) this approach has been coupled with the standard Richardson-Lucy (RL) algorithm. However, their way of dealing with negative values of the single-image is not the most appropriate one. In this note we propose a different method which provides better reconstructions and convergence rates.
\end{abstract}

Key words. methods: data analysis - methods: statistical - techniques: image processing

\section{Introduction}

An important activity in image processing is the multipleimage deblurring, i.e., the restoration of a set of observed images of a fixed object when each image is deteriorated by a different spatially invariant point spread function (PSF).

Suppose one has $p$ observed images, $\rho_{j}(x, y), j=$ $1,2, \ldots, p$, of a fixed two-dimensional object $f_{0}(x, y)$, each of which is degraded by a different spatially invariant blurring operator. That is,

$\rho_{j}(x, y)=\iint K_{j}\left(x-x^{\prime}, y-y^{\prime}\right) f_{0}\left(x^{\prime}, y^{\prime}\right) \mathrm{d} x^{\prime} \mathrm{d} y^{\prime}+b_{j}(x, y)$,

where, for each $j, K_{j}(x, y)$ is a space invariant PSF and $b_{j}(x, y)$ is a function describing the background due to sky emission. The image restoration problem of interest is to find an estimate $f(x, y)$ of $f_{0}(x, y)$, given the observed images $\left\{\rho_{j}(x, y)\right\}_{j=1}^{p}$, the PSFs $\left\{K_{j}(x, y)\right\}_{j=1}^{p}$, and an estimate of $b_{j}(x, y)$.

In practice, the experimental images are discrete $N \times N$ arrays of pixels (for simplicity, we assume square images). Therefore model (1) has to be recast in the form

$\rho_{j}(m, n)=\sum_{m^{\prime}, n^{\prime}=0}^{N-1} K_{j}\left(m-m^{\prime}, n-n^{\prime}\right) f_{0}\left(m^{\prime}, n^{\prime}\right)+b_{j}(m, n)$.
As it is well known, in many situations Eq. (2) can be well approximated by cyclic convolution. This permits rewriting it as

$\widehat{\rho}_{j}(m, n)=\widehat{K}_{j}(m, n) \widehat{f}_{0}(m, n)+\widehat{b}_{j}(m, n)$,

where the symbol " - " denotes the Discrete Fourier Transform (DFT).

Because of independent statistics of photon arrivals from the object and the background, the observed image is given by

$$
\begin{aligned}
g_{j}(m, n) & =\text { Poisson }\left[\left(K_{j} * f_{0}\right)(m, n)\right]+\text { Poisson }\left[b_{j}(m, n)\right], \\
& =g_{j}^{(o)}(m, n)+g_{j}^{(b)}(m, n),
\end{aligned}
$$

where the operator Poisson $[\lambda]$ denotes the realization of a Poissonian process with mean value $\lambda$. The two terms correspond to the photons from the object and the background, respectively.

A maximum likelihood iterative algorithm, the socalled Ordered-Subset/Expectation-Maximization algorithm (OS/EM), has been proposed by Bertero \& Boccacci (2000). It can be considered a multiple-image extension of the classic Richardson-Lucy (RL) algorithm and represents one of the most efficient techniques presently available. However, since each iteration requires the computation of $4 p$ DFTs and typically some hundreds of iterations are necessary before convergence, the computational burden remains rather heavy. A further problem with methods like OS/EM is that, in order to avoid 
bottlenecks in the memory traffic that can slow down computation, all the $p$ images have to be stored in the fast memory. In general, such memory has a limited capacity and this can be troublesome for large images.

\section{The single-image approach}

In the context of a blurring model with additive Gaussian noise, Vio et al. (2004) suggest an efficient approach where the images $g_{j}(m, n)$ are composed together in a single image $\zeta(m, n)$ that is equivalent, in the least-squares sense, to the original set of data. Apart from the computational efficiency and storage memory, this has also the benefit that no new software is required since standard packages can be used.

The PSF and the image $\widehat{\zeta}(m, n)$ of this single-image approach are given by

$$
\begin{aligned}
& \widehat{K}_{j_{0}}(m, n)=\max \left[\widehat{K}_{1}(m, n), \widehat{K}_{2}(m, n), \ldots, \widehat{K}_{p}(m, n)\right], \\
& \widehat{\zeta}(m, n)=\frac{\widehat{g}_{j_{0}}(m, n)+\sum_{j \neq j_{0}}\left[\widehat{K}_{j}^{*}(m, n) / \widehat{K}_{j_{0}}^{*}(m, n)\right] \widehat{g}_{j}(m, n)}{1+\sum_{j \neq j_{0}}\left[\left|\widehat{K}_{j}(m, n)\right|^{2} /\left|\widehat{K}_{j_{0}}(m, n)\right|^{2}\right]} .
\end{aligned}
$$

In Eq. (5), the operator $\max [\cdot]$ extracts the element in the array with the largest modulus, and $j_{0}$ is the value of the corresponding index $j$.

From Eq. (4) it follows that $\widehat{\zeta}(m, n)$ can be decomposed into the object and background contribution

$\widehat{\zeta}(m, n)=\widehat{\zeta}^{(o)}(m, n)+\widehat{\zeta}^{(b)}(m, n)$.

Thanks to the linearity of the relationship between $\widehat{\zeta}(m, n)$ and the images $\widehat{g}_{j}(m, n)$, whose expected values are given by Eq. (3), it easy to show that the expected value of $\widehat{\zeta}(m, n)$ is given by

$\widehat{\rho}(m, n)=\widehat{K}_{j_{0}}(m, n) \widehat{f_{0}}(m, n)+\widehat{b}(m, n)$,

the relationship between $\widehat{b}(m, n)$ and $\widehat{b}_{j}(m, n)$ being given by Eq. (6). If all the backgrounds $b_{j}(m, n)$ are constant, they contribute only to $\widehat{b}_{j}(0,0)$. Consequently, by assuming that all the PSFs are normalized to unit volume, so that $\widehat{K}_{j}(0,0)=1$, it follows that the average backgound of $\zeta(m, n)$ is just the arithmetic mean of the different backgrounds

$b(m, n)=\frac{1}{p} \sum_{j=1}^{p} b_{j}(m, n)$.

When the backgrounds are not constant, the situation is more difficult. However, a simple solution consists of subtracting the background from $g_{j}(m, n)$ before the computation of $\zeta(m, n)$.

A problem, not so serious for the LS algorithms but that potentially can prevent the convergence of algorithms such as RL, is that $\zeta(m, n)$, as well as $K_{j_{0}}(m, n)$, may have negative values. In this case, there is no guarantee that in the RL iteration

$\boldsymbol{f}^{(k+1)}=\boldsymbol{f}^{(k)} \odot \mathcal{A}^{T} \frac{\zeta}{\mathcal{A} \boldsymbol{f}^{(k)}+\boldsymbol{b}}$,

$\boldsymbol{f}^{(k+1)}$ is always non-negative, a condition necessary for the convergence. Here, $\boldsymbol{\zeta}, \boldsymbol{f}$ and $\boldsymbol{b}$ are arrays obtained by column stacking $\zeta(m, n), f(m, n)$ and $b(m, n), \mathcal{A}$ is the block-circulant with circulant-blocks (BCCB) matrix corresponding to the PSF $K_{j_{0}}(m, n)$, " $\odot$ " denotes Hadamard (component-wise) multiplication, and the fraction of two vectors has to be intended as a Hadamard division. In general, these negative values are significant only for small values of $p$, and become rapidly less important when the number of images increases. A possible solution for this problem is a careful analysis of the derivation of the original RL algorithm (e.g., Lucy 1974). In fact, the PSF appears only within integrals. Therefore, if these integrals remain positive despite the PSF not being everywhere non-negative, then the iterations still work. A simple way to obtain such a condition is to modify model (10) to

$\boldsymbol{f}^{(k+1)}=\boldsymbol{f}^{(k)} \odot \mathcal{A}^{T} \frac{\zeta+\boldsymbol{b}^{*}}{\mathcal{A} \boldsymbol{f}^{(k)}+\boldsymbol{b}+\boldsymbol{b}^{*}}$,

where $\boldsymbol{b}^{*}=\beta \times \operatorname{ABS}\left[\min \left(\zeta_{\min }, 0\right)\right], \beta$ is a real, positive scalar, and $\zeta_{\min }$ is the minimum in the image $\zeta$. Although it is very difficult to define the smallest value of $\beta$ that allows the convergence of algorithm (11), in all the numerical experiments carried out with $\beta=1$ (see below) we have never experienced convergence failure. However, situations cannot be excluded that require larger values for this parameter. Given the wellknown optimal properties of the RL algorithm also when no a priori assumption is made on the specific noise model, this operation has to be expected not to deteriorate the quality of the results.

A possible alternative, adopted by Anconelli et al. (2005), consists of zeroing the negative values in $\zeta(m, n)$ and in the corresponding PSF. However, such a procedure introduces spurious discontinuities (i.e., high frequency components) which make the resulting single-image and the corresponding PSF incompatible with the blurring model given by Eqs. (2), (4). For this reason, a deterioration of the performance has to be expected for both the convergence rate of the algorithms and the quality of the restoration.

All these points are confirmed by our numerical experiments. In particular, Figs. 1 and 2 show the results obtainable in the case of a subject with a sharp outline superimposed on a sky whose intensity, in the blurred image, is set to $1 \%$ of the maximum value of the image. Three images are available to have a single-image where the negative values are not negligible. The PSFs are bidimensional Gaussian with dispersion along the major axis set to twelve pixels, and to four pixels along the minor axis. Their inclinations take the values $0^{\circ}-60^{\circ}-120^{\circ}$. Each image is contaminated with Poissonian noise. A peak signal to noise ratio $(S N R)^{1}$ equal to $40 \mathrm{~dB}$ has been adopted. This experiment corresponds to a difficult problem. In fact, the sharp features of the subject imply that the high frequencies in its spectrum (i.e., those more contaminated by the noise) are important. Moreover, the PSFs have a symmetry quite different from the circular one and this exacerbates the problem of the negative values in $\zeta(m, n)$ and in the corresponding PSF. Four deblurring methods have been used: the single-image RL method (11), the classic RL algorithm applied to the versions

\footnotetext{
${ }^{1}$ Here $S N R=20 \log \left(g_{\max } / g_{\max }^{1 / 2}\right) \mathrm{dB}$, where $g_{\max }$ is the maximum value for all the images $g_{j}(m, n)$.
} 


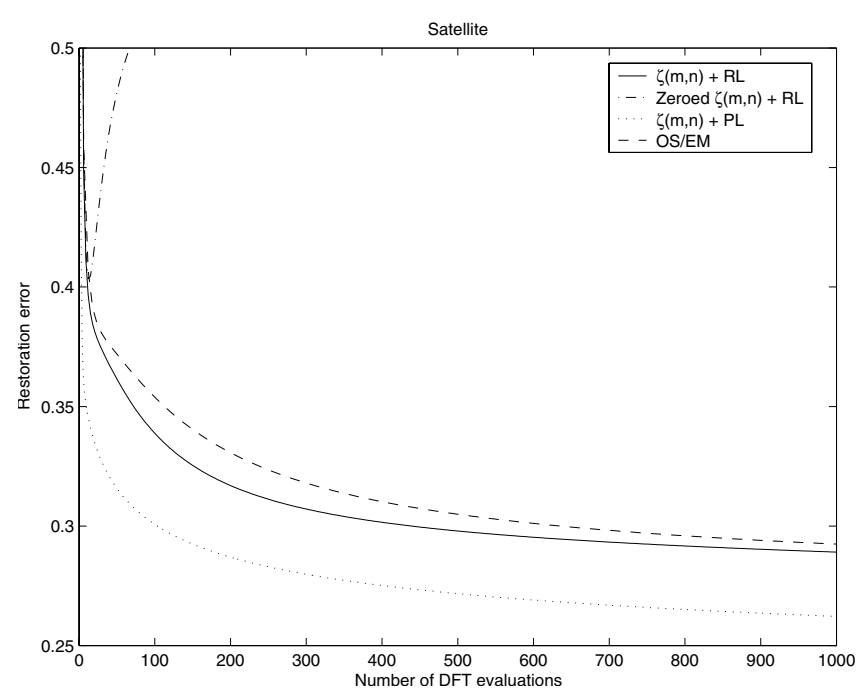

Fig. 1. Restoration error $\left\|\boldsymbol{f}^{(k)}-\boldsymbol{f}_{0}\right\| /\left\|\boldsymbol{f}_{0}\right\|$ vs. the number of DFT evaluations for the algorithms described in the text. Here, the subject of interest is the classic satellite outline shown in Fig. 2. The size of the image is $256 \times 256$ pixels. Peak $S N R=40 \mathrm{~dB}$.
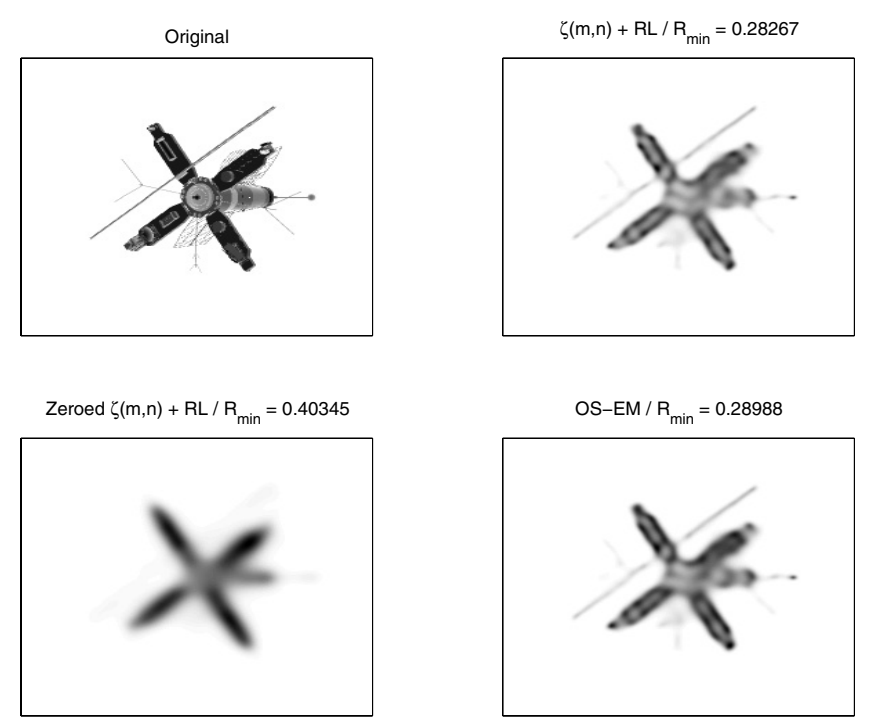

Fig. 2. The original satellite image and the corresponding results obtained with the deblurring methods described in the text. $R_{\min }$ provides the smallest restoration error obtained during the iteration of the algorithm.

of $\zeta(m, n)$ and in the corresponding PSF with the negative values set to zero, a classic LS Projected-Landweber (PL) method applied to $\zeta$, and the OS/EM method. From this experiment it is evident that: a) in general, OS/EM and the single-image RL methods provide quite similar results, with the latter presenting a faster convergence rate; $b$ ) the zeroing of the negative values in $\zeta(m, n)$ and in the corresponding PSF is a harmful operation; c) the PL algorithm is able to provide excellent results. The situation does not change if different values of SNR are used as well as different values of the background.

The above results are confirmed by the more realistic experiment shown in Figs. 3 and 4. Here, the subject of interest is a diffuse object and the PSFs correspond to the ideal ones for

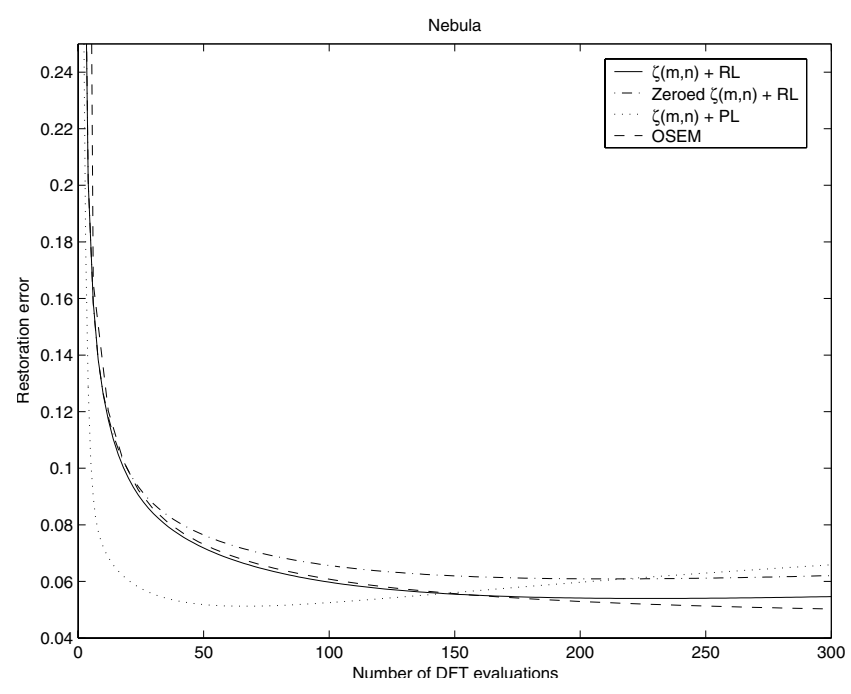

Fig. 3. Restoration error $\left\|\boldsymbol{f}^{(k)}-\boldsymbol{f}_{0}\right\| /\left\|\boldsymbol{f}_{0}\right\|$ vs. the number of DFT evaluations for the algorithms described in the text. Here, the subject of interest is the nebula shown in Fig. 4. The size of the image is $128 \times 128$ pixels. Peak $S N R=40 \mathrm{~dB}$.
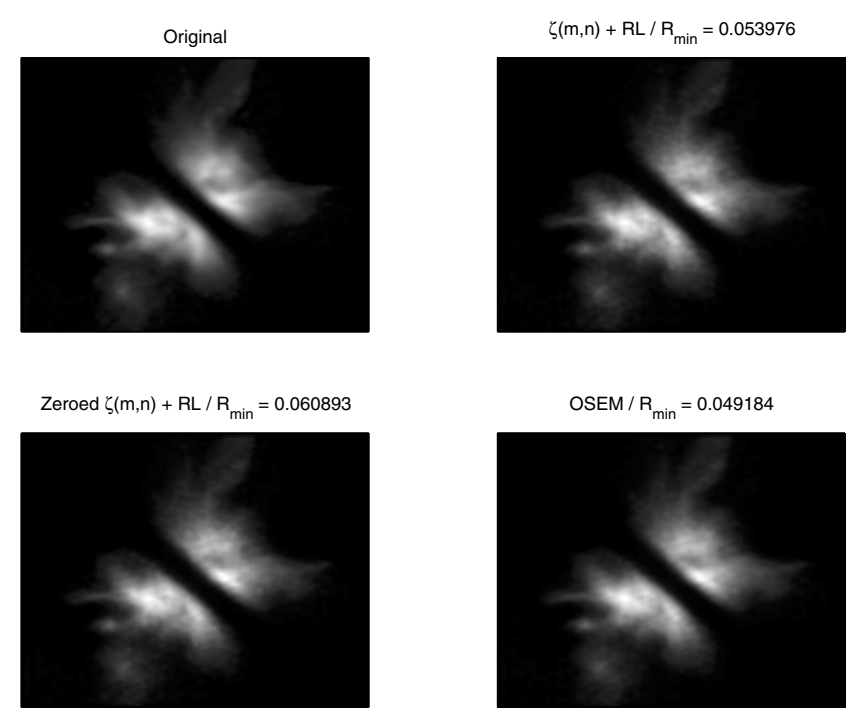

Fig. 4. The original nebula image and the corresponding results obtained with the deblurring methods described in the text. $R_{\min }$ provides the smallest restoration error obtained during the iteration of the algorithm.
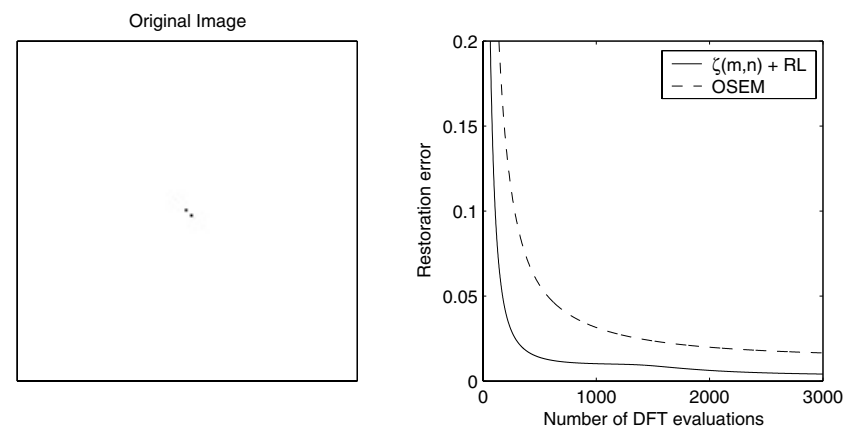

Fig. 5. Restoration error vs. the number of DFT evaluations for the algorithms described in the text. Here, the subject of interest is a close binary system whose components have the same intensity. The restoration error is calculated only on the two pixels containing the stars. The size of the image is $128 \times 128$ pixels. Peak $S N R=40 \mathrm{~dB}$. 
LBT (see Bertero \& Boccacci 2000). The other conditions are identical to those in the previous experiment.

The superiority of the single-image approach is suggested also by Fig. 5 that shows the results obtainable in the case of a close binary system. The simulation has been carried out under the same conditions used for the diffuse object experiment.

This result disagrees with the conclusions of Anconelli et al. (2005). This is not unexpected since, as claimed above, the zeroing operation is not correct. In their numerical experiments Anconelli et al. (2005) used a slightly modified version of $\zeta(m, n)$. However, our simulations show that the two singleimages provide almost identical results.

So far we have assumed that the level of the contaminating noise is the same in all the images. The extension of the method when this condition is not satisfied is straightforward. It is sufficient to compute $\zeta(m, n)$ by means of Eqs. (5) and (6) with $g_{j}(m, n) \longrightarrow w_{j} g_{j}(m, n)$ and $K_{j}(m, n) \longrightarrow w_{j} K_{j}(m, n)$, where $w_{j}$ is a weight related to the $S N R$ of the $j$ th image (e.g., see Vio et al. 2004). The rest of the procedure remains unaltered.

\section{Summary}

We have considered the problem of restoring a set of images of a fixed object blurred by different space-invariant PSFs and
Poissonian noise. Although the currently available multipleimage methods are able to provide results of satisfactory quality, they are computationally expensive and demanding of storage memory. This is because they work cyclically on one image at a time. We have shown that, after minor adjustments, the classic RL algorithm coupled with the single-image approach, developed by Vio et al. (2004) in the LS context, represents an advantageous choice. Apart from the obvious substantial savings in storage memory and the possibility to implement it by using standard, currently available numerical algorithms, the convergence rate is often superior and the quality of the restoration is not expected to deteriorate.

Acknowledgements. We thank Prof. M. Bertero for the PSFs of LBT.

\section{References}

Anconelli, B., Bertero, M., Boccacci, B., Carbillet, M., \& Lanteri, H. 2005, A\&A, 430, 731

Angel, J. R. P., Hill, J. M., Strittmatter, P. A., Salinari, P., \& Weigelt, G. 1998, in Astronomical Interferometry, ed., R. D. Reasenberg, Proc. SPIE, 3352, 881

Bertero, M., \& Boccacci, P. 2000, A\&A, 147, 323

Lucy, L. B. 1974, AJ, 79, 745

Vio, R., Nagy, J., Tenorio, L., et al. 2004, A\&A, 416, 403 